Mappemonde

Revue trimestrielle sur l'image géographique et les formes du territoire

$131 \mid 2021$

Varia

\title{
Un flipbook pour représenter des mobilités
}

Anne-Christine Bronner et Françoise Bahoken

\section{OpenEdition}

Journals

Édition électronique

URL : https://journals.openedition.org/mappemonde/6320

DOI : 10.4000/mappemonde.6320

ISSN : 1769-7298

Éditeur

UMR ESPACE

\section{Référence électronique}

Anne-Christine Bronner et Françoise Bahoken, « Un flipbook pour représenter des mobilités »,

Mappemonde [En ligne], 131 | 2021, mis en ligne le 08 juillet 2021, consulté le 14 juillet 2021. URL : http://journals.openedition.org/mappemonde/6320; DOI : https://doi.org/10.4000/mappemonde 6320

Ce document a été généré automatiquement le 14 juillet 2021.

\section{(c) (†) (ㅇ)}

La revue Mappemonde est mise à disposition selon les termes de la Licence Creative Commons Attribution - Pas d'Utilisation Commerciale - Partage dans les Mêmes Conditions 4.0 International. 


\title{
Un flipbook pour représenter des mobilités
}

\author{
Anne-Christine Bronner et Françoise Bahoken
}

1 La question de la carte animée dans le cadre du Mapathon du colloque Tous (im)mobiles, tous cartographes? (cartomob.sciencesconf.org) a été traitée de façon originale par la réalisation d'un flipbook par Maxime Salles, du collectif les Artisans Cartographes.

2 Le collectif les Artisans Cartographes est féru des approches cartographiques originales. Il propose des représentations inédites du territoire alliant les savoirs et savoir-faire du ou de la cartographe-géomaticien-ne, notamment de solides connaissances dans la collecte et la mobilisation de la donnée géographique, les méthodes et outils des Systèmes d'Information Géographiques (SIG), à une démarche artisanale de création et de confection de la carte. Citons, sans être exhaustives, leurs jeux de cartes à jouer sur Nantes, Lyon, Le Havre ; leurs mappzles et autres cartes à déguster ; la carte En 2017, tous les chemins mènent au Havre qui représente finement les tracés routiers menant au Havre sous la forme d'un chevelu hydrographique... Ils initient également le fanzine Mercator pour honorer la vivacité actuelle de la production cartographique francophone.

3 Cette contribution est portée par Maximes Salles. Elle prend la forme d'un flipbook, c'est-à-dire d'un petit livre formé d'une collection d'images successives, que l'on visualise en la feuilletant avec le pouce pour créer une animation.

4 Maxime Salles décrit sa proposition en ces termes: "Le nombre de passagers transportés de et vers la Corse varie fortement en fonction des saisons. Pour représenter cette saisonnalité, j'ai choisi d'utiliser le flipbook. D'un coup de pouce agile, la Corse s'anime. Les points proportionnels grossissent et rapetissent donnant l'impression que le cœur de la Corse bat au rythme des saisons. »

On comprend dès lors pourquoi le jury du Mapathon a été séduit par le recours à juste titre au procédé du flipbook pour représenter des mouvements aériens. La carte résulte, quel que soit le contexte de sa réalisation, d'une série de choix.

7 Le choix, ici, est de fabriquer un objet artisanal, réalisé à la main, pour maîtriser l'animation d'un phénomène dynamique: le flipbook. C'est aussi celui d'une 
visualisation réalisée avec la technique classique du stop motion utilisée pour certains dessins animés ou films d'animation, c'est-à-dire la succession d'images statiques nécessaires à la perception d'un mouvement.

Le choix du territoire représenté répond à l'objectif de porter un message cartographique simple et clair : la forme de la Corse isolée sur un fond bleu se reconnaît aisément. Sur le plan thématique, la saisonnalité des navettes aériennes fait varier significativement le nombre de passagers sur les quatre aéroports mentionnés. Les cartes ont été créées avec arcGIS.

Le choix du traitement cartographique des données (sous arcGIS) s'est fondé sur leur dimension temporelle, avec une attention portée au choix d'un pas de temps mensuel pour agréger le nombre de passagers pour la période 2010-2019 et créer la succession de cartes. Le «calibrage » de la taille des cercles proportionnels et le jeu des couleurs avec un choix harmonieux des différents tons ont pour objectif de bien différencier le fond et forme, le contenant et le contenu.

Figure 1. Copie écran du flipbook

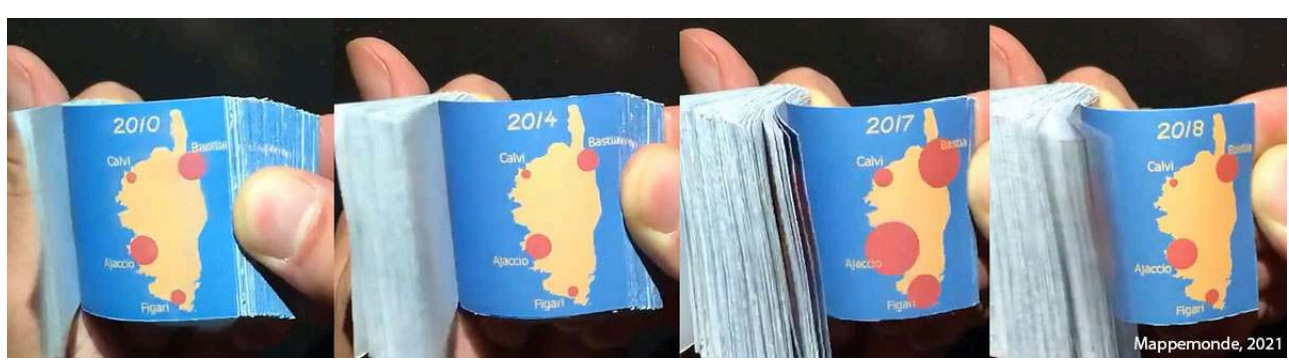

Réalisé par Maxime Salles (collectif Artisans cartographes)

L'impression, le découpage et l'assemblage manuel finalisent l'objet cartographique. C'est l'étape du choix du papier et de son épaisseur, pour assurer le caractère opérationnel du flipbook, qui passe également par la maîtrise d'un bon cutter et de la patience, une imprimante à jet d'encre et un stock de cartouches.

11 La cartographie, dont la définition officielle identifie qu'elle est à la fois une science, une technique et un art se retrouve pleinement dans la démarche proposée par les artisans cartographes, avec cette attention portée à la création d'objets à manipuler (flipbook, pliages...) et un soin particulier porté à la qualité des productions (jeux de cartes à jouer, sérigraphie ou impression offset...).

C'est là un autre pan de la science reproductible et ouverte, celle qui permet à tout un chacun de fabriquer des cartes, y compris dans le cadre d'une conception artisanale.

Ce média ne peut être affiché ici. Veuillez vous reporter à l'édition en ligne http:// journals.openedition.org/mappemonde/6320 